\title{
Towards Relativistic Skyrmions
}

\author{
Marcus A. C. Torres* \\ Instituto de Física, \\ Universidade Federal do Rio de Janeiro, \\ Caixa Postal 68528, \\ 21941-972 Rio de Janeiro, RJ, Brasil. \\ E-mail: mtorreseif.ufrj.br
}

Henrique Boschi-Filho, Nelson R. F. Braga

Instituto de Física,

Universidade Federal do Rio de Janeiro,

Caixa Postal 68528,

21941-972 Rio de Janeiro, RJ, Brasil.

Email: boschi@if.ufrj.br, bragaeif.ufrj.br

MATTHIAS IHL

School of Theoretical Physics,

Dublin Institute for Advanced Studies,

10 Burlington $R d$,

Dublin 4, Ireland.

[0.3cm] Email: msihlestp.dias.ie

\begin{abstract}
We revisit baryons in the Skyrme model. Starting from static baryons in the helicity eigenstates, we generalize their wavefunctions to the non-static and relativistic regime. A new representation for gamma matrices in the soliton collective space is constructed and the corresponding Dirac equation is obtained. As an example, we draw consideration on how to apply this new representation on the calculus of vector current vacuum expectation values for baryon states of spin and isospin half and arbitrary momenta and we show how elastic form factors can be derived.
\end{abstract}

7th Conference Mathematical Methods in Physics - Londrina 2012,

16 to 20 April 2012

Rio de Janeiro, Brazil

${ }^{*}$ Speaker. 


\section{Introduction}

Skyrmions are solitons, classical, static solution from NL $\sigma \mathrm{M}$ of pions [1] and they are identified as baryons in such model. It is a model with chiral Lagrangean and spontaneous chiral symm breaking. It has attracted renewed interest as a simplified description of baryons in the large $N_{c}$ limit of QCD. Here we review the work presented in [2] where we show a simple way to overcome its static or slow moving condition, extending it to a relativistic framework.

The static properties of baryons in the Skyrme model (skyrmions) have been studied in [3] , where baryonic quantum states emerge from a canonical quantization of the soliton moduli. In [4], Sakai and Sugimoto derive the Skyrme model as the $3+1$ dimensional pion effective theory descending from the dynamics of the gauge fields living on flavor $D 8$-branes that probe the $D 4$ brane geometry generated by a stack of color branes.

Here, after introducing the Skyrme model and the soliton canonical quantization in section 2, we calculate relativistic baryon wave functions in their helicity eigenstates (section 3) generalizing the results of [3] by boosting wave functions in the momentum direction. We show that the state representation is in a form compatible with the Dirac spinor representation. In section 4, as an example we show how to obtain form factors from the Lorentz tensorial decomposition of matrix elements of baryonic currents in our relativistic approach.

\section{Skyrme Model}

Skyrmions are soliton solutions of a nonlinear effective field theory of pions. The action of the Skyrme model is given by

$$
S=\int d^{4} x\left(\frac{f_{\pi}^{2}}{4} \operatorname{tr}\left(U^{-1} \partial_{\mu} U\right)^{2}+\frac{1}{32 e^{2}} \operatorname{tr}\left[U^{-1} \partial_{\mu} U, U^{-1} \partial_{\nu} U\right]^{2}\right)
$$

where the pion fields $\pi\left(x^{\mu}\right)$ are encoded in the SU(2) valued Skyrme field,

$$
\left.U\left(x^{\mu}\right)=\frac{\sigma\left(x^{\mu}\right) \mathbb{I}+\pi^{i}\left(x^{\mu}\right) \cdot \tau^{i}}{f_{\pi}}=e^{i \pi\left(x^{\mu}\right) / f_{\pi}} \text { (weak pion field limit }\right),
$$

where $\sigma^{2}\left(x^{\mu}\right)+\vec{\pi}^{2}\left(x^{\mu}\right)=f_{\pi}^{2}$. U transforms as $U\left(x^{\mu}\right) \rightarrow g_{L} U\left(x^{\mu}\right) g_{R}^{-1}$ under the chiral symmetry $S U(2)_{L} \times S U(2)_{R}$ present in the Lagrangian.

The Skyrmion is a static solution that goes to unity value at infinity breaking chiral symmetry to the diagonal subgroup. In this case $\mathrm{U}$ becomes a map

$$
U: \mathbb{R}^{3}+\{\infty\}=S^{3} \rightarrow S U(2) \equiv S^{3}
$$

and a topological charge $n_{B} \in \pi_{3}(S U(2))=\mathbb{Z}$ is identified with the baryon number.

\subsection{SU(2) collective coordinates}

We begin by reviewing the moduli space approximation method to quantize Skyrmions. The fundamental idea [3] is to realize that there is a moduli of soliton solution given by $S U(2)$ rotations of a given one, $U_{0}$, and define a slowly moving soliton by a time dependent rotation,

$$
U\left(t, x^{M}\right)=V U_{0}\left(x^{M}, X^{M}(t)\right) V^{-1},
$$


where $V=V\left(t, x^{M}\right)$ is an $S U(2)$ element and $X^{M}, M=\{1, \ldots, 3\}$, represents the position of the soliton in the spatial $\mathbb{R}^{3}$. Introducing the collective coordinates $\boldsymbol{a}(t)=a_{4}(t)+i a_{a}(t) \tau^{a}$ as a point in $S^{3}$ representing the $S U(2)$ orientation, we note $V(t, x) \rightarrow \boldsymbol{a}(t)$ as $x \rightarrow \infty$.

When $\boldsymbol{a}(t) U_{o} \boldsymbol{a}^{-1}(t)$ is replaced in the original Lagrangian, a new Lagrangian emerge,

$$
L=-M+2 \lambda \sum_{i=1}^{4}\left(\dot{a}_{i}\right)^{2}
$$

Introducing conjugate momenta $\pi_{i}=\partial L / \partial \dot{a}_{i}=-i \frac{\partial}{\partial a_{i}}$, we find the Hamiltonian:

$$
H=M+\lambda \nabla_{S^{3}}^{2}
$$

The eigenstates (wavefunctions) are factorized into radial and spherical harmonics components. On the $S^{3}$, they are scalar spherical harmonics and are known to be homogenous polynomials

$$
T^{(l)}\left(a_{I}\right)=C_{I_{1} \cdots I_{l}} a_{I_{1}} \cdots a_{I_{l}},
$$

where $C_{I_{1} \cdots I_{l}}$ is a traceless symmetric tensor of rank $l$.

The dimension of the tensor $C$ and therefore the dimension of the space of spherical harmonics of degree $l, \mathbb{H}_{l}$ is $(l+1)^{2}$. The space $\mathbb{H}_{l}$ is a representation of the rotation group $S O(4)$ and corresponds to the $\left(S_{l / 2}, S_{l / 2}\right)$ representation of $(S U(2) \times S U(2)) / \mathbb{Z}_{2} \simeq S O(4) . S_{l / 2}$ denotes the $\operatorname{spin} l / 2$ representation of $S U(2)$, with $\operatorname{dim} S_{l / 2}=l+1$. Under group rotation $\boldsymbol{a}$ transforms as

$$
\boldsymbol{a} \rightarrow g_{I} \boldsymbol{a} g_{J}, \quad g_{I, J} \in S U(2)_{I, J} .
$$

where $S U(2)_{I}$ and $S U(2)_{J}$ are identified with the isospin rotation and the spatial rotation, respectively.

\section{Static and Relativistic Baryons}

The collective state is quantized considering slowly moving solitons. We will extend it to relativistic baryons by simply treating them as static solitons boosted in a given direction. First, we review the spherical harmonics tensors to static nucleons. The lowest states are at $l=1$ and the tensors become linear in $a_{I}$ coordinates. They correspond to states with spin and isospin $1 / 2$ and we identify them with protons and neutrons. In spinorial notation we write the particle states as

$$
|N, h\rangle=\chi^{N} \otimes \chi_{h}=: \chi_{h}^{N}
$$

where $N=\{p, n\}, h=\{+,-\}$ and

$$
\chi^{p}=\chi_{+}=\left(\begin{array}{l}
1 \\
0
\end{array}\right) \quad, \quad \chi^{n}=\chi_{-}=\left(\begin{array}{l}
0 \\
1
\end{array}\right) .
$$

The isospin $I_{3}$ and spin $J_{3}$ operators in this representation read

$$
I_{a}=\frac{i}{2}\left(a_{4} \frac{\partial}{\partial a_{a}}-a_{a} \frac{\partial}{\partial a_{4}}-\varepsilon_{a b c} a_{b} \frac{\partial}{\partial a_{c}}\right), J_{a}=\frac{i}{2}\left(-a_{4} \frac{\partial}{\partial a_{a}}+a_{a} \frac{\partial}{\partial a_{4}}-\varepsilon_{a b c} a_{b} \frac{\partial}{\partial a_{c}}\right),
$$

and their eigenstates are given by

$$
|p,+\rangle=\frac{a_{1}+i a_{2}}{\pi},|p,-\rangle=-\frac{i\left(a_{4}-i a_{3}\right)}{\pi},|n,+\rangle=\frac{i\left(a_{4}+i a_{3}\right)}{\pi},|n,-\rangle=-\frac{\left(a_{1}-i a_{2}\right)}{\pi} .
$$




\subsection{Nucleon relativistic wavefunctions}

In generalizing the expressions aboveto the relativistic case, we initially restrict the discussion to proton and neutron states separately and disregard the isospin information. We introduce a relativistic spinor for a fermion with a given momentum $\vec{p}$,

$$
u(p, h)=\frac{1}{\sqrt{2 E}}\left(\begin{array}{c}
f \chi_{h} \\
\frac{\vec{p} \cdot \vec{\sigma}}{f} \chi_{h}
\end{array}\right), \text { with } f=\sqrt{E+m_{B}}
$$

Here $\chi_{h}$ is a helicity eigenstate, which means $\vec{p} \cdot \vec{\sigma} \chi_{h}=h|\vec{p}| \chi_{h}$ where $h= \pm 1$. This yields the eigenstates

$$
\chi_{+}(\vec{p})=\frac{1}{\sqrt{2|\vec{p}|\left(|\vec{p}|+p_{3}\right)}}\left(\begin{array}{c}
|\vec{p}|+p_{3} \\
p_{1}+i p_{2}
\end{array}\right) \quad, \quad \chi_{-}(\vec{p})=\frac{1}{\sqrt{2|\vec{p}|\left(|\vec{p}|+p_{3}\right)}}\left(\begin{array}{c}
-p_{1}+i p_{2} \\
|\vec{p}|+p_{3}
\end{array}\right) .
$$

Notice that $\chi_{h}^{\dagger} \chi_{h}=1$ and $|\vec{p}|=\sqrt{p_{1}^{2}+p_{2}^{2}+p_{3}^{3}}$. Hence we expect the proton and neutron linear $(l=1)$ spherical harmonic tensors (equivalent to $\chi_{h}(\vec{p})$ helicity eigenstates) to be

$\chi_{+}^{p}\left(a_{i} \cdot \vec{p}\right)=\frac{\left(|\vec{p}|+p_{3}\right)\left(a_{1}+i a_{2}\right)-i\left(p_{1}+i p_{2}\right)\left(a_{4}-i a_{3}\right)}{\pi \sqrt{2|\vec{p}|\left(|\vec{p}|+p_{3}\right)},}, \chi_{-}^{p}\left(a_{i} \cdot \vec{p}\right)=\frac{\left(-p_{1}+i p_{2}\right)\left(a_{1}+i a_{2}\right)-i\left(p_{3}+|\vec{p}|\right)\left(a_{4}-i a_{3}\right)}{\pi \sqrt{2|\vec{p}|\left(|\vec{p}|+p_{3}\right)}}$,
$\chi_{+}^{n}\left(a_{i} \cdot \vec{p}\right)=\frac{\left(|\vec{p}|+p_{3}\right) i\left(a_{4}+i a_{3}\right)-\left(p_{1}+i p_{2}\right)\left(a_{1}-i a_{2}\right)}{\pi \sqrt{2|\vec{p}|\left(|\vec{p}|+p_{3}\right)}}, \chi_{-}^{n}\left(a_{i} \cdot \vec{p}\right)=\frac{\left(-p_{1}+i p_{2}\right) i\left(a_{4}+i a_{3}\right)-\left(p_{3}+|\vec{p}|\right)\left(a_{1}-i a_{2}\right)}{\pi \sqrt{2|\vec{p}|\left(|\vec{p}|+p_{3}\right)}}$.

The expression above is not valid for $p_{1}=p_{2}=0$ and $p_{3}=-p$ and in that case we recall that helicity changes sign when momentum changes in sign to the opposite direction,

$$
\chi_{h}^{N}\left(a_{i},-\vec{p}\right) \equiv \chi_{-h}^{N}\left(a_{i}, \vec{p}\right)
$$

After some algebra we can verify that (3.7) are eigenfunctions of $\vec{p} . \vec{J}$ operators (3.3):

$$
\vec{p} . \vec{J} \chi_{+}^{N}\left(a_{i}, \vec{p}\right)=+\frac{1}{2}|\vec{p}| \chi_{+}^{N}\left(a_{i}, \vec{p}\right) \quad \text { and } \quad \vec{p} . \vec{J} \chi_{-}^{N}\left(a_{i}, \vec{p}\right)=-\frac{1}{2}|\vec{p}| \chi_{-}^{N}\left(a_{i}, \vec{p}\right) .
$$

Similarly to Dirac 4-spinors (3.5), The relativistic nucleon SU(2) wavefunctions are

$$
u_{N}\left(a_{i}, \vec{p},+\right)=\frac{1}{\sqrt{2 E}}\left(\begin{array}{c}
f \chi_{+}^{N}\left(a_{i}, \vec{p}\right) \\
\frac{|\vec{p}|}{f} \chi_{+}^{N}\left(a_{i}, \vec{p}\right)
\end{array}\right) \quad, \quad u_{N}\left(a_{i}, \vec{p},-\right)=\frac{1}{\sqrt{2 E}}\left(\begin{array}{c}
f \chi_{-}^{N}\left(a_{i}, \vec{p}\right) \\
-\frac{|\vec{p}|}{f} \chi_{-}^{N}\left(a_{i}, \vec{p}\right)
\end{array}\right) .
$$

The explicit dependence on $a_{I} \in S^{3}$ is not directly observable and the integration in $S^{3}$ moduli recovers the 4D Dirac spinor with appropriate isospin.

\subsection{Dirac equation and spin sum}

In order to work with relativistic $S U(2)$ wavefunctions instead of Dirac spinorial notation, we define the substitutes of gamma matrices in the $S U(2)$ collective space by simply replacing the Pauli matrices $\sigma^{i}$ with $2 J^{i}$ operators (3.3). Hence, the new $2 \times 2$ gamma matrices are

$$
\gamma^{0}=-i\left(\begin{array}{cc}
1 & 0 \\
0 & -1
\end{array}\right) \quad, \quad \gamma^{i}=-i\left(\begin{array}{cc}
0 & 2 J^{i} \\
-2 J^{i} & 0
\end{array}\right) \text {. }
$$


Such operators act only on spin and we will disregard the isospin index for now. Upon such substitution we can verify the validity of the Dirac equation:

$$
\begin{aligned}
\left(i \not p+m_{B}\right) u_{N}\left(a_{i}, \vec{p}, h\right) & =0 . \\
\bar{u}_{N}\left(a_{i}, \vec{p}, h\right)\left(i \not p+m_{B}\right) & =0 .
\end{aligned}
$$

Since $J^{i}$ operators have real eigenvalues and behave like Pauli matrices, we define their operation to the left by transpose conjugation: $\psi_{h}^{\dagger}(\vec{p} \cdot \vec{J})=\left(\vec{p} \cdot \vec{J} \psi_{h}\right)^{\dagger}=|\vec{p}| \frac{h}{2} \psi_{h}^{\dagger}$.

As mentioned before, the spinor normalization is given by an integration of the $a_{i}$ moduli,

$$
\bar{u}\left(\vec{p}, h^{\prime}\right) u(\vec{p}, h)=\int_{S^{3}} \bar{u}\left(a_{i}, \vec{p}, h^{\prime}\right) u\left(a_{i}, \vec{p}, h\right)
$$

Working out the integrand,

$$
\begin{aligned}
\bar{u}\left(a_{i}, \vec{p}, h^{\prime}\right) u\left(a_{i}, \vec{p}, h\right) & =\frac{1}{2 E}\left(f \chi_{h^{\prime}}^{*}\left(a_{i}, \vec{p}\right) \quad \frac{|\vec{p}|}{f} h^{\prime} \chi_{h^{\prime}}^{*}\left(a_{i}, \vec{p}\right)\right)\left(\begin{array}{cc}
1 & 0 \\
0 & -1
\end{array}\right)\left(\begin{array}{c}
f \chi_{h}^{a_{i}}(\vec{p}) \\
\frac{|\vec{p}|}{f} h \chi_{h}\left(a_{i}, \vec{p}\right)
\end{array}\right) \\
& =\frac{1}{2 E}\left(f^{2}-h^{\prime} h \frac{|\vec{p}|^{2}}{f^{2}}\right) \chi_{h^{\prime}}^{*}\left(a_{i}, \vec{p}\right) \chi_{h}\left(a_{i}, \vec{p}\right)
\end{aligned}
$$

In order to integrate (3.15) over $S^{3}$ we write $\left(a_{1}, a_{2}, a_{3}, a_{4}\right)$ in spherical coordinates:

$$
a_{1}=\sin \theta_{0} \sin \theta_{1} \sin \theta_{2}, a_{2}=\sin \theta_{0} \sin \theta_{1} \cos \theta_{2}, a_{3}=\sin \theta_{0} \cos \theta_{1}, a_{4}=\cos \theta_{0} .
$$

The volume element is $d \Omega_{3}=\sin ^{2} \theta_{0} \sin \theta_{1} d \theta_{0} d \theta_{1} d \theta_{2}$. Using (3.7), the integration over (3.15) turns out to be

$$
\int_{S^{3}} \bar{u}\left(a_{i}, \vec{p}, h^{\prime}\right) u\left(a_{i}, \vec{p}, h\right)=\frac{1}{2 E}\left(\left(E+m_{B}\right)-h^{\prime} h\left(E-m_{B}\right)\right) \int_{S^{3}} \chi_{h^{\prime}}^{a_{i} *}(\vec{p}) \chi_{h}^{a_{i}}(\vec{p})=\frac{m_{B}}{E} \delta_{h^{\prime} h} .
$$

The spin sum is given by

$$
\sum_{h} u(\vec{p}, h) \bar{u}(\vec{p}, h)=\sum_{h} \int_{S^{3}} u\left(a_{i}, \vec{p}, h\right) \bar{u}\left(a_{i}, \vec{p}, h\right),
$$

where the integrand of (3.17) reads

$$
\begin{aligned}
& u\left(a_{i}, \vec{p}, h\right) \bar{u}\left(a_{i}, \vec{p}, h\right)=\frac{1}{2 E}\left(\begin{array}{c}
f \chi_{h}^{a_{i}}(\vec{p}) \\
\frac{2 \vec{J} \cdot \vec{p}}{f} \chi_{h}^{a_{i}}(\vec{p})
\end{array}\right)\left(f \chi_{h}^{a_{i} *}(\vec{p})-\frac{2 \vec{J} \cdot \vec{p}}{f} \chi_{h}^{a_{i} *}(\vec{p})\right) \\
& =\frac{1}{2 E}\left(\begin{array}{cc}
E+m_{B} & -2 \vec{J} \cdot \vec{p} \\
2 \vec{J} \cdot \vec{p} & -E+m_{B}
\end{array}\right)\left|\chi_{h}^{a_{i}}(\vec{p})\right|^{2}=\frac{1}{2 E}\left(-i \not p+m_{B}\right)\left|\chi_{h}^{a_{i}}(\vec{p})\right|^{2} .
\end{aligned}
$$

Integrating over (3.18) we get the spin sum (3.17),

$$
\sum_{h} \int_{S^{3}} u\left(a_{i}, \vec{p}, h\right) \bar{u}\left(a_{i}, \vec{p}, h\right)=\frac{1}{2 E}\left(-i \not p+m_{B}\right) \sum_{h} \int_{S^{3}}\left|\chi_{h}^{a_{i}}(\vec{p})\right|^{2}=\frac{1}{E}\left(-i \not p+m_{B}\right) .
$$




\section{Application: elastic form factors}

The $\mathrm{SU}(2)$ vector current integrated over the unit sphere of spatial $\mathbb{R}^{3}[3]$ behave as

$$
\int d \Omega^{2} J_{V}^{0, c} \sim \operatorname{Tr}\left[\left(\partial_{0} \boldsymbol{a}\right) \boldsymbol{a}^{-1} \tau^{c}\right], \quad \text { and } \quad \varepsilon^{i j k} \int d \Omega^{2} x^{j} J_{V}^{k, c} \sim \operatorname{Tr}\left[\tau^{i} \boldsymbol{a}^{-1} \tau^{c} \boldsymbol{a}\right],
$$

where it should be noted that the $S U(2)_{V}$ currents were decomposed as $J_{V}^{\mu}=J_{V}^{\mu, c} \tau^{c}$. Since the collective coordinates do not depend on the position in $\mathbb{R}^{3}$, general vector currents read

$$
J_{V}^{0, c}(\vec{k})=e^{-i \vec{k} \cdot \vec{X}} I_{c}, J_{V}^{i, c}(\vec{k})=i e^{-i \vec{k} \cdot \vec{X}} \Lambda \varepsilon_{i j a} q_{j} \operatorname{Tr}\left(\tau^{c} \boldsymbol{a} \tau^{a} \boldsymbol{a}^{-1}\right)
$$

where $q_{j}:=p_{j}^{\prime}-p_{j}$. When calculating the vector current proton-proton matrix elements, we are only interested in the $c=3$ components (see above). We define the Dirac and Pauli form factors according to the following decomposition of current matrix elements,

$\left\langle p_{X}, B_{X}, s_{X}\left|J_{V}^{\mu, a}(0)\right| p, B, s\right\rangle=\frac{i\left(\tau^{a}\right)_{I_{3}^{X} I_{3}}}{2(2 \pi)^{3}}\left(\eta^{\mu v}-\frac{q^{\mu} q^{v}}{q^{2}}\right) \bar{u}\left(p_{X}, s_{X}\right)\left[\gamma_{v} F_{B B_{X}}^{D, a}\left(q^{2}\right)+\kappa_{B} \sigma_{v \lambda} q^{\lambda} F_{B B_{X}}^{P, a}\left(q^{2}\right)\right] u(p, s)$

In the elastic case, in the Breit frame, with $\vec{p}=-\vec{p}^{\prime}=-\frac{q}{2} \hat{z}$, the expression (4.3) becomes

$$
\left\langle p_{X}, B_{X}, s_{X}\left|J_{V}^{0, a}(0)\right| p, B, s\right\rangle=\frac{1}{2(2 \pi)^{3}}\left(\tau^{a}\right)_{I_{3}^{X} I_{3}}\left(\frac{m_{B}}{E}\right) \delta_{s_{X},-s}\left[F_{B}^{D, a}\left(q^{2}\right)-\frac{q^{2}}{4 m_{B}^{2}} F_{B}^{P, a}\left(q^{2}\right)\right]
$$

$$
\left\langle p_{X}, B_{X}, s_{X}\left|J_{V}^{i, a}(0)\right| p, B, s\right\rangle=-\frac{1}{2(2 \pi)^{3}}\left(\tau^{a}\right)_{I_{3}^{X} I_{3}}\left(\frac{i}{2 E}\right) \varepsilon^{i j k} q_{j}\left(\sigma_{k}\right)_{s_{X},-s}\left[F_{B}^{D, a}\left(q^{2}\right)+F_{B}^{P, a}\left(q^{2}\right)\right]
$$

The equations above contain a relativistic correction not present in Skyrme Models.

We need to calculate the vevs of $\operatorname{Tr}\left(\tau^{a} \boldsymbol{a}^{-1} \tau^{3} \boldsymbol{a}\right)$ in (4.2) in order to find the form factors.

In spherical coordinates the traces become $(a=3)$ :

$$
\operatorname{Tr}\left(\tau^{3} \boldsymbol{a}^{-1} \tau^{3} \boldsymbol{a}\right)=2\left(\cos \left(\theta_{0}\right)^{2}+\cos \left(2 \theta_{1}\right) \sin \left(\theta_{0}\right)^{2}\right) .
$$

The general result for baryon states $(\mathrm{a}=1,2,3)$ is

$$
\begin{gathered}
\left\langle\operatorname{Tr}\left(\tau^{a} \boldsymbol{a}^{-1} \tau^{3} \boldsymbol{a}\right)\right\rangle \equiv\left\langle B_{X}, \vec{p}^{\prime}, h^{\prime}\left|\operatorname{Tr}\left(\tau^{a} \boldsymbol{a}^{-1} \tau^{3} \boldsymbol{a}\right)\right| B_{0}, \vec{p}, h\right\rangle= \\
\int_{S^{3}} \Omega^{3} \bar{u}_{X}\left(a_{i}, \vec{p}^{\prime}, h^{\prime}\right) \operatorname{Tr}\left(\tau^{a} \boldsymbol{a}^{-1} \tau^{3} \boldsymbol{a}\right) u_{0}\left(a_{i}, \vec{p}, h\right)=\frac{-1}{3 \sqrt{E_{X} E}}\left(f f_{X}-\frac{h h^{\prime}|\vec{p}|\left|\vec{p}^{\prime}\right|}{f f_{X}}\right) \chi_{h^{\prime}}^{\dagger}\left(\vec{p}^{\prime}\right) \sigma^{a} \chi_{h}(\vec{p})
\end{gathered}
$$

where $f_{X}=\sqrt{E_{X}+m_{B_{X}}}$ and $\chi_{h^{\prime}}\left(\vec{p}^{\prime}\right)$ and $\chi_{h}(\vec{p})$ are the helicity spinors (3.6). The equation above plays an important role in the calculation of the electromagnetic proton form factors [4]. In the elastic case, in Breit frame, $\vec{p}=-\vec{p}^{\prime}=-\frac{q}{2} \hat{z}$ and (4.7) takes the form

$$
\left\langle\operatorname{Tr}\left(\tau^{a} \boldsymbol{a}^{-1} \tau^{3} \boldsymbol{a}\right)\right\rangle=\frac{\left(E+m_{B}-h^{\prime} h\left(E-m_{B}\right)\right)}{2 E}\left(-\frac{2}{3}\right) \sigma_{h^{\prime},-h}^{a}=\left\{\begin{array}{l}
-\frac{2}{3} \frac{m_{B}}{E} \sigma_{h^{\prime},-h}^{1} \text { for } a=1 . \\
-\frac{2}{3} \frac{m_{B}}{E} \sigma_{h^{\prime},-h}^{2} \text { for } a=2 . \\
-\frac{2}{3} \sigma_{h^{\prime},-h}^{3} \text { for } a=3 .
\end{array}\right.
$$

where we used the substitution (3.8). This amounts to a relativistic correction in the direction of movement $\hat{z}$ w.r.t. the static case [3], of a factor of $\frac{m_{B}}{E}$. 
Using (4.8) in the vector currents (4.2), the current matrix elements can be written as

$$
\begin{aligned}
& \left\langle\frac{q}{2} \hat{z}, B_{X}, h^{\prime}\left|J_{V}^{0,3}(0)\right|-\frac{q}{2} \hat{z}, B, h\right\rangle=\frac{1}{2(2 \pi)^{3}}\left(\frac{m_{B}}{E}\right) \delta_{-h^{\prime} h} \\
& \left\langle\frac{q}{2} \hat{z}, B_{X}, h^{\prime}\left|J_{V}^{i, 3}(0)\right|-\frac{q}{2} \hat{z}, B, h\right\rangle=-\frac{1}{2(2 \pi)^{3}}\left(\frac{i}{2 E}\right) \frac{8 m_{B}}{3} \Lambda \varepsilon_{i 3 a} q_{3} \sigma_{-h^{\prime} h}^{a}
\end{aligned}
$$

where we utilized eq. (3.16) and noted that $\left\langle 2 I^{3}\right\rangle_{+1 / 2,+1 / 2}=\left(\tau^{3}\right)_{+1 / 2,+1 / 2}=1$ for the proton-proton matrix elements. Employing eqs. (4.9), (4.10) and eqs. (4.4), (4.5) we arrive at

$$
F_{B}^{D, 3}\left(q^{2}\right)=\left(1+\frac{q^{2}}{4 m_{B}^{2}}\right)^{-1}\left(\frac{2}{3} \frac{\Lambda q^{2}}{m_{B}}+1\right), \quad F_{B}^{P, 3}\left(q^{2}\right)=\left(1+\frac{q^{2}}{4 m_{B}^{2}}\right)^{-1}\left(\frac{8}{3} m_{B} \Lambda-1\right) .
$$

They are proton form factors for $\tau^{3}$ isovector current .

\section{Conclusion}

Here we presented a relativistic generalization for non-static baryons in Skyrme models. The correct relativistic normalization for currents and the vacuum expectation values appears quite naturally in our approach.

\section{Acknowledgements}

The authors H.B-F., N.R.F.B. and M.A.C.T. are supported by CAPES and CNPq (Brazilian research agencies). The work of M.I. was supported by an IRCSET postdoctoral fellowship.

\section{References}

[1] T. H. R. Skyrme, “A Nonlinear field theory,” Proc. Roy. Soc. Lond. A 260, 127 (1961).

[2] H. Boschi-Filho, N. R. F. Braga, M. Ihl and M. A. C. Torres, Phys. Rev. D 85, 085013 (2012) [arXiv:1111.2287 [hep-th]].

[3] G. S. Adkins, C. R. Nappi, E. Witten, "Static Properties of Nucleons in the Skyrme Model," Nucl. Phys. B228, 552 (1983).

[4] T. Sakai and S. Sugimoto, "Low energy hadron physics in holographic QCD," Prog. Theor. Phys. 113, 843 (2005) [arXiv:hep-th/0412141].

[5] K. Hashimoto, T. Sakai and S. Sugimoto, "Holographic Baryons: Static Properties and Form Factors from Gauge/String Duality,” Prog. Theor. Phys. 120 (2008) 1093 [arXiv:0806.3122 [hep-th]]. 